\title{
Three new species of Mycena sect. Longisetae
}

\section{Aravindakshan DM and Manimohan P}

\author{
Department of Botany, University of Calicut, Kerala, 673 635, India
}

Aravindakshan DM, Manimohan P 2014 - Three new species of Mycena sect. Longisetae. Mycosphere 5(2), 290-297, Doi 10.5943/mycosphere/5/2/3

\begin{abstract}
Three new species of the agaric genus Mycena (M. lomamaya, M. jatila and M. pelava) are described from Kerala State, India and are assigned to sect. Longisetae. Comprehensive descriptions, photographs, and comparisons with phenetically similar species are provided. A key to the species of Mycena sect. Longisetae known from Kerala is given.
\end{abstract}

Key words - Agaricales - Basidiomycota - biodiversity - Mycenaceae - taxonomy

\section{Introduction}

Mycena sect. Longisetae A.H. Smith ex Maas G., originally established by Maas Geesteranus (1983) as a monotypic taxon based on M. longiseta Höhn., was redefined by Desjardin and Horak (2002) and Desjardin et al. (2002) to accommodate eleven species worldwide: $M$. aciculata (A.H. Sm.) Desjardin \& E. Horak, M. breviseta Höhn., M. brevisetosa Corner, M. brunneisetosa Corner, M. clavulifera (Berk. \& Broome) Sacc., M. indica Manim. \& Leelav. (current name: Mycena lomavritha Manim.), M. khonkhem Desjardin et al., M. longiseta Höhn., M. palmicola Desjardin et al., M. tenuisetosa Corner and M. trichocephala Singer. All included species of sect. Longisetae have several long, needle-like, thick-walled hairs or pileosetae on the pileus at least in the primordial stage (Desjardin et al. 2002). In addition, these species have ascending, narrowly adnate to free lamellae, a pruinose to hispid stipe with a typically discoid base, ellipsoid basidiospores, 4-spored basidia, a hymenium devoid of pleurocystidia, a pileus margin often beset with cystidia, dextrinoid pileal and stipe trama, smooth caulocystidia that typically taper towards the apex, a pileipellis composed of either acanthocysts or spinulose hyphae and a stipitipellis with non-spinulose hyphae (Desjardin et al. 2002, Aravindakshan \& Manimohan 2011). Subsequently, Boonpratuang (2009) tentatively placed $M$. variicystis Boonprat., a new species from Thailand, in this section but Aravindakshan \& Manimohan (2013) included that species in sect. Spinosae Aravind. \& Manim. This was followed by Aravindakshan \& Manimohan (2011) who assigned both a new species that they described from India (M. saloma Aravind. \& Manim.) and a Brazilian species (M. rhaphidocephala Maas G. \& de Meijer) originally placed by Maas Geesteranus \& de Meijer (1998) in sect. Sacchariferae to sect. Longisetae. Southern Asia seems to be the centre of diversity of this group (Desjardin et al. 2002, Aravindakshan \& Manimohan 2011).

During the course of our studies on the genus Mycena in Kerala State, India, we came across three hitherto undescribed species that also belong to sect. Longisetae and these species are described, illustrated and discussed here. 


\section{Materials \& Methods}

Microscopic observations were made on material stained with $1 \%$ aqueous solution of Congo red and mounted in 3\% aqueous $\mathrm{KOH}$. Melzer's reagent was used to observe whether the spores and tissues were amyloid. For evaluation of the range of spore size, twenty basidiospores from each specimen cited were measured. Colour codes used in the description are from Kornerup \& Wanscher (1978). The examined collections are deposited at Kew (Mycology) Herbarium and the Kew accession numbers (e.g., K(M)178340) are indicated. The concept of Desjardin et al. (2002) is followed for Mycena sect. Longisetae.

\section{Results and Discussion}

\section{Taxonomy}

Mycena lomamaya Aravind. \& Manim., sp. nov. Fig. $1 \mathrm{a}-\mathrm{j}$ MycoBank MB 804818

Etymology - lomamaya (Sanskrit), beset with hairs.

Characterised by brownish grey pileus with fine hairs; pseudocollariate lamellae; discoid stipe base; ellipsoid to subcylindrical and weakly amyloid basidiospores; cheilocystidia and pileus marginal cells with long, cylindrical excrescences; slightly gelatinous pileipellis hyphae with large, subglobose protrusions as well as short, cylindrical excrescences all over; and morphologically similar pileosetae and caulocystidia. Differing from Mycena aciculata in having amyloid basidiospores, pileus marginal cells and a different type of cheilocystidia with long, cylindrical excrescences.

Basidiomata very small, delicate. Pileus $1-4.5 \mathrm{~mm}$ diam., $0.5-1 \mathrm{~mm}$ high, convex when young, becoming hemispherical with age; surface brownish grey (6D2) at the centre and on the striations, whitish towards margin, and brownish grey (6C2) elsewhere, finely hairy, pellucidstriate, finely pruinose; margin straight, entire when young, becoming undulate with age. Lamellae pseudocollariate, whitish, up to $0.5 \mathrm{~mm}$ wide, subdistant, with lamellulae of 1-2 lengths; edge finely torn under a lens, concolorous with the sides. Stipe $5-22.5 \times 0.25-0.5 \mathrm{~mm}$, central, terete, almost equal, hollow; surface translucent, white, finely pubescent, more so towards the base, almost glabrous at the apex; base discoid, thin, with finely radiating basal mycelium. Context narrow, concolorous with the pileus surface. Odour and taste not distinctive.

Basidiospores (5) 7-9 (10) $\times(3) 3.5-4.5$ (5) $(8.025 \pm 0.617 \times 3.975 \pm 0.42) \mu \mathrm{m}, \mathrm{Q}=1.56-$ $2.5, \mathrm{Qm}=2.03$, ellipsoid, subcylindrical or rarely subamygdaliform, thin-walled, hyaline, smooth, with a few refractive guttules, weakly amyloid. Basidia 11-19 $\times 7.5-10 \mu \mathrm{m}$, clavate, sometimes subpedicellate, hyaline, bearing 4 sterigmata up to $5 \mu \mathrm{m}$ long. Lamella-edge sterile. Cheilocystidia crowded, 13.5-30 × 7-11 $\mathrm{m}$, clavate, sometimes subpedicellate thin-walled, hyaline, with short or long cylindrical excrescences $(1-20 \times 1-1.5 \mu \mathrm{m})$ on the upper half. Pleurocystidia absent. Lamellar trama regular to subregular; hyphae 3-23 $\mu \mathrm{m}$, thin-walled, hyaline, vinoid in Melzer's reagent. Subhymenium pseudoparenchymatous. Pileus trama subregular or interwoven; hyphae 10.5-26.5 (30) $\mu \mathrm{m}$ wide, thin-walled, hyaline, vinoid in Melzer's reagent. Pileipellis a cutis; hyphae 1.5-3 $\mu \mathrm{m}$ wide, slightly gelatinized, thin-walled, hyaline, with intercalary subglobose protrusions, entirely covered with cylindrical excrescences $(1-3 \times 0.5-1 \mu \mathrm{m})$. Hypodermium distinct, composed of 3.5$10 \mu \mathrm{m}$ wide, thin-walled, hyaline, smooth hyphae. Pileosetae 43-207.5 $\times 5-25 \mu \mathrm{m}$, aculeate, thickwalled $(2-5.5 \mu \mathrm{m})$, hyaline, smooth. Pileus marginal cells 11-31.5 ×6-11 $\mu \mathrm{m}$, clavate, obovoid or cylindrical, thin-walled, hyaline, with cylindrical excrescences $(1-17 \times 1-2 \mu \mathrm{m})$. Stipitipellis a cutis; hyphae 2-5.5 $\mu \mathrm{m}$ wide, thin-walled, hyaline, smooth. Caulocystidia 65-203 $\times 5-14 \mu \mathrm{m}$, aculeate, thick-walled $(1-5 \mu \mathrm{m})$, hyaline, rarely branching. Stipe trama moderately vinoid in Melzer's reagent. Clamp connections seen on all hyphae.

On decaying leaves, scattered or in groups, August.

Distribution - known only from the type locality. 


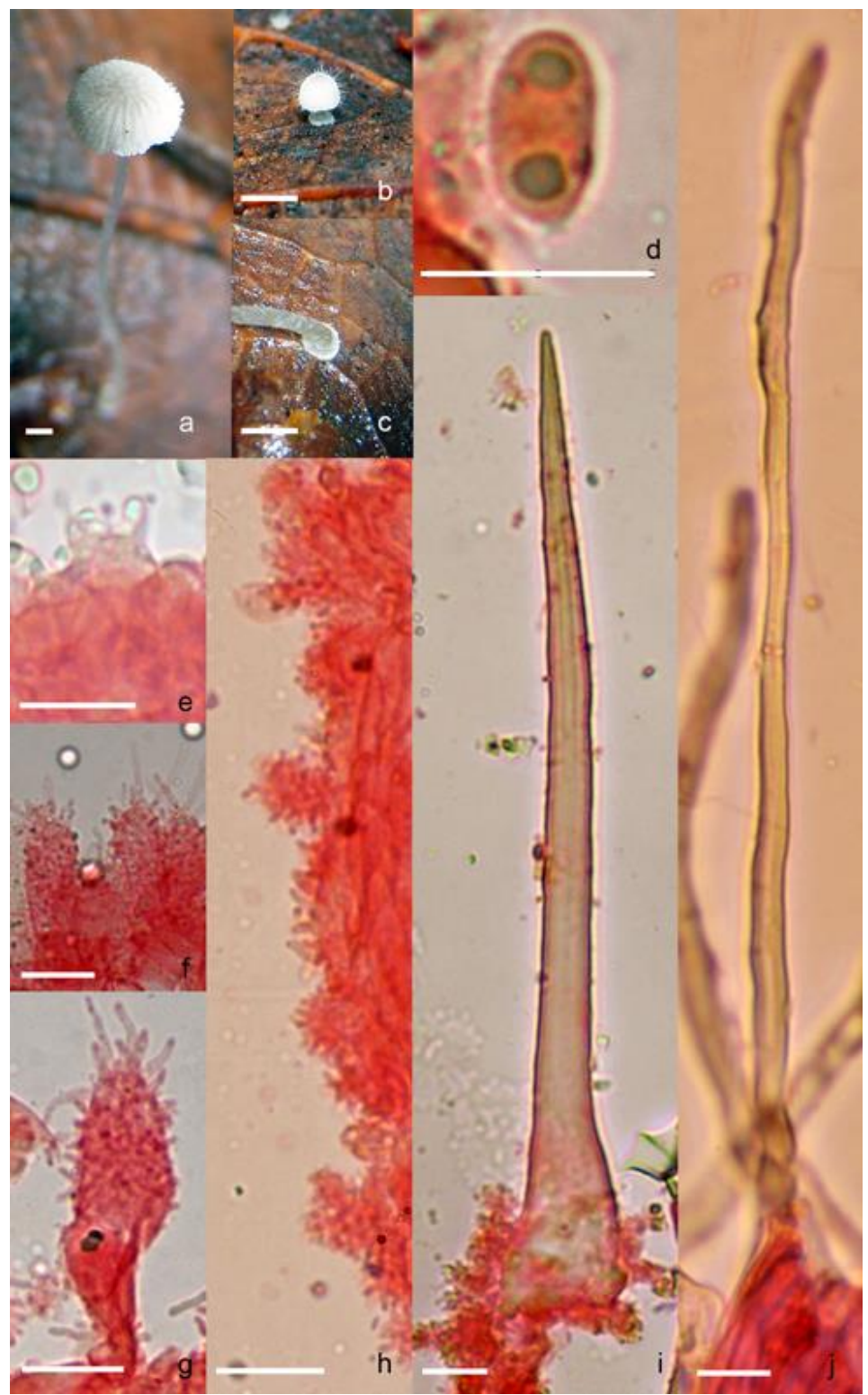

Fig. 1 a-j-Mycena lomamaya. a, Mature basidioma. b, Young basidioma. c, Discoid base of stipe. d, Basidiospore. e, Basidium. f, Cheilocystidia. g, Pileus marginal cell. h, Pileipellis. i, Pileoseta. j, Caulocystidium. - Bars $=1 \mathrm{~mm}$ for basidiomata and $10 \mu \mathrm{m}$ for micro-structures.

Material examined - India, Kerala State, Idukki District, Anamudi Shola National Park, 17 August 2010, D.M. Aravindakshan, K(M) 178340 (K-holotype).

Notes - The key to the worldwide members of Mycena sect. Longisetae (Desjardin et al. 2002), does not lead the present species to any of the eleven species treated. The nearest species seems to be $M$. aciculata, a common temperate Northern Hemisphere species, because of the similar-sized basidiospores, cheilocystidia, and pileosetae (up to $200 \mu \mathrm{m}$ long), thick-walled, setoid caulocystidia, and gelatinized pileipellis tissues. But $M$. aciculata differs in having inamyloid basidiospores, different type of cheilocystidia (fusoid or clavate with 1-3 necks), and in lacking pileus marginal cells. Mycena khonkhem is similar in having greyish pileus; lamellae that are often pseudocollariate, relatively narrow basidiospores (3.5-4.5 $\mu \mathrm{m}$ diam.), foliicolous substrate, acanthocystoid cheilocystidia and pileus marginal cystidia, a gelatinized, cutis-type pileipellis of densely spinulose hyphae and relatively short hyaline pileosetae; but that species lacks caulocystidia, the long, cylindrical protrusions on both cheilocystidia and pileus marginal cystidia and the large subglobose protrusions on pileipellis hyphae that are characteristic of M. lomamaya. For a comparison with other species known from Kerala, refer to the Key given below. 
Etymology - jatila (Sanskrit), hairy.

Characterised by minute basidiomata with pale brownish pileus disc and discoid stipe base; ellipsoid and inamyloid basidiospores; ellipsoid or fusoid cheilocystidia with a beak-like apical protrusion; gelatinized pileipellis hyphae; slightly thick-walled, short, obclavate pileosetae sometimes with excrescences at the base; obclavate or fusoid pileus marginal cells; and caulocystidia with a long, flexuous and gradually tapering apical part. Differing from Mycena aciculata in having smaller basidiomata, smaller basidiospores, shorter pileosetae, smaller cheilocystidia and thin-walled caulocystidia.

Basidiomata very small, delicate. Pileus $0.5-1 \mathrm{~mm}$ diam., initially hemispherical, becoming applanate with age; surface pale brown (5C3) at the centre and on the striations, whitish elsewhere, translucent to sulcate-striate, with very fine hairs; margin straight and entire when young, becoming plane and finely torn. Lamellae 7-9 reaching the stipe, free, white, less than $0.5 \mathrm{~mm}$ wide, subdistant; lamellulae absent or rarely in one length; edge finely torn under a lens, concolorous with the sides. Stipe 3-8 $\times 0.25-0.5 \mathrm{~mm}$, central, terete, tapering towards the apex, hollow; surface translucent, white, with very fine, white pubescence, more so towards base, almost glabrous at the apex; base discoid. Context narrow, concolorous with the pileus surface. Odour and taste not recorded.

Basidiospores 6-7.5 × 3-4 (6.825 $\pm 0.568 \times 3.55 \pm 0.426) \mu \mathrm{m}, \mathrm{Q}=1.71-2.33, \mathrm{Qm}=1.94$, ellipsoid to subcylindrical, thin-walled, hyaline, smooth, inamyloid. Basidia 13-17 $\times 6.5-8.5 \mu \mathrm{m}$, clavate, hyaline, bearing 4 sterigmata up to $3 \mu \mathrm{m}$ long. Lamella-edge fertile. Cheilocystidia scattered, 9-15.5 $\times 3.5-7 \mu \mathrm{m}$, ellipsoid or fusoid with an apical beak-like protrusion $(5-13 \mu \mathrm{m}$ long), smooth, thin-walled. Pleurocystidia none. Lamellar trama subregular; hyphae 5-19 $\mu \mathrm{m}$, thinwalled, hyaline, faintly vinoid in Melzer's reagent. Subhymenium inconspicuous. Pileus trama subregular; hyphae 8-22 $\mu \mathrm{m}$ wide, thin-walled, hyaline, faintly vinoid in Melzer's reagent. Pileipellis a cutis of hyphae with terminal pileosetae; hyphae $2-11 \mu \mathrm{m}$ wide, embedded in a gelatinous matter, thin-walled, hyaline, with short, conic or cylindrical excrescences $(0.5-4 \times 0.5-$ $1.5 \mu \mathrm{m})$. Pileosetae $46-111 \times 9-33 \mu \mathrm{m}$, obclavate with a tapering apex, slightly $(0.5-1 \mu \mathrm{m})$ thickwalled, sometimes with a few small protrusions $(5-9 \times 4-7 \mu \mathrm{m})$ and sparse excrescences $(1-3 \times$ $0.5-1 \mu \mathrm{m})$ at the base. Pileus marginal cells $39-67 \times 6-0 \mu \mathrm{m}$, obclavate to fusoid-ventricose with a long tapering apical part, thin-walled, hyaline, smooth. Stipitipellis a cutis; hyphae 1-3.5 $\mu \mathrm{m}$ wide, thin-walled, hyaline, smooth. Caulocystidia $18.5-113 \times 5-11.5 \mu \mathrm{m}$, with a swollen base and a long, flexuous, gradually tapering apex, thin-walled, hyaline, smooth. Stipe trama vinoid in Melzer's reagent. Clamp connections seen on all hyphae except at the base of cheilocystidia.

On decaying leaves, solitary or scattered, September.

Distribution - known only from the type locality.

Material examined - India, Kerala State, Kollam District, Sasthamkotta, 29 September 2010, D. M. Aravindakshan, K(M) 178341 (K-holotype).

Notes - The key to the worldwide species of Mycena sect. Longisetae (Desjardin et al. 2002) does not lead the present species to any particular one of the eleven species treated in it. However characters such as a cutis-type pileipellis composed of repent, densely spinulose hyphae and pileosetae that are less than $200 \mu \mathrm{m}$ long lead to a cluster of four species: M. aciculata, M. brevisetosa, M. khonkhem and M. palmicola. Mycena aciculata has additional similarity to M. jatila in having inamyloid basidiospores, somewhat similar type of cheilocystidia and gelatinized pileipellis hyphae. But that species differs in having somewhat larger basidiomata, larger basidiospores $(7.5-10.5 \times 3.8-4.7 \mu \mathrm{m}$ ), longer pileosetae (up to $200 \mu \mathrm{m}$ ) larger cheilocystidia and thick-walled caulocystidia. Mycena brevisetosa differs in having pileosetae up to $350 \mu \mathrm{m}$ long, amyloid basidiospores with mean width of approximately $5 \mu \mathrm{m}$ and in growing on bark. Mycena khonkhem differs from $M$. jatila mainly in having acanthocystoid cheilocystidia and pileus marginal cystidia and amyloid basidiospores and in lacking caulocystidia. Like $M$. jatila, $M$. palmicola also have pileus less than $1 \mathrm{~mm}$ in diam. However, habitat on palm leaves, amyloid basidiospores and absence of cheilocystidia makes the latter species different from M. jatila. 


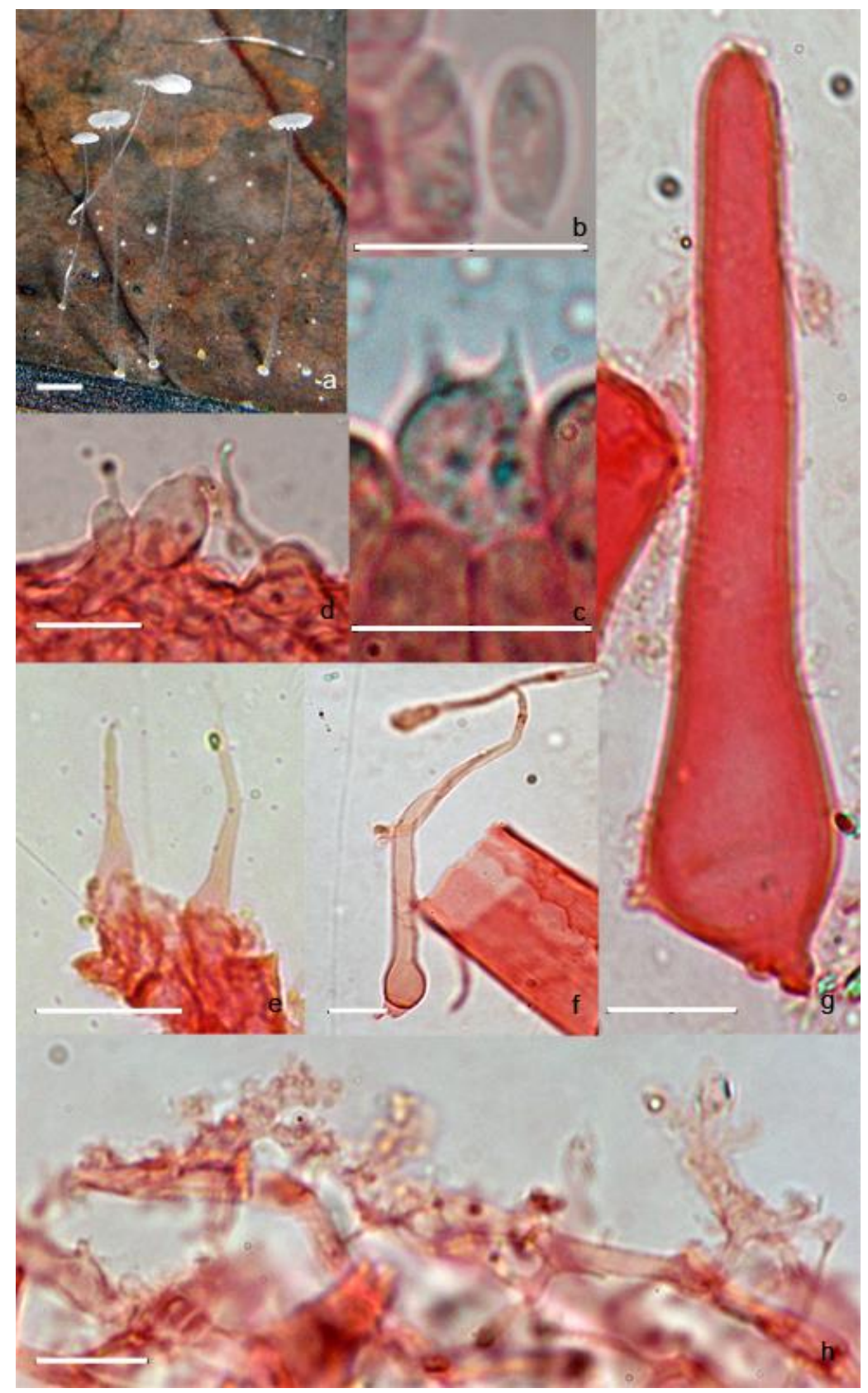

Fig. 2 a-h - Mycena jatila. a, Basidiomata. b, Basidiospore. c, Basidium. d, Cheilocystidia. e, Pileus marginal cells. $\mathrm{f}$, Caulocystidium. g, Pileoseta. h, Hyphae of the pileipellis. - Bars $=1 \mathrm{~mm}$ for basidioma and $10 \mu \mathrm{m}$ for micro-structures.

Mycena pelava Aravind. \& Manim., sp. nov.

Fig. $2 \mathrm{a}-\mathrm{h}$

MycoBank MB 804820

Etymology - pelava (Sanskrit), delicate.

Characterised by minute foliicolous basidiomata; pale greyish pileus with hyaline pileosetae; swollen stipe base with radiating basal mycelium; ellipsoid to subcylindrical and weakly amyloid basidiospores; cylindrical and smooth cheilocystidia; aculeate pileosetae with subglobose or geniculate base; and smooth, thin-walled, fusoid caulocystidia with a long tapering apex. Differing from Mycena longiseta in having cheilocystidia and much shorter pileosetae.

Basidiomata very small, delicate. Pileus $0.5-1 \mathrm{~mm}$ diam., up to $0.5 \mathrm{~mm}$ high, convex to broadly convex with a central shallow depression; surface pale grey (1B1) at the centre and on the striations, whitish elsewhere, initially translucent-striate, becoming faintly sulcate-striate, beset with very fine hairs; margin straight, crenate when young, becoming undulate or fringed. Lamellae 


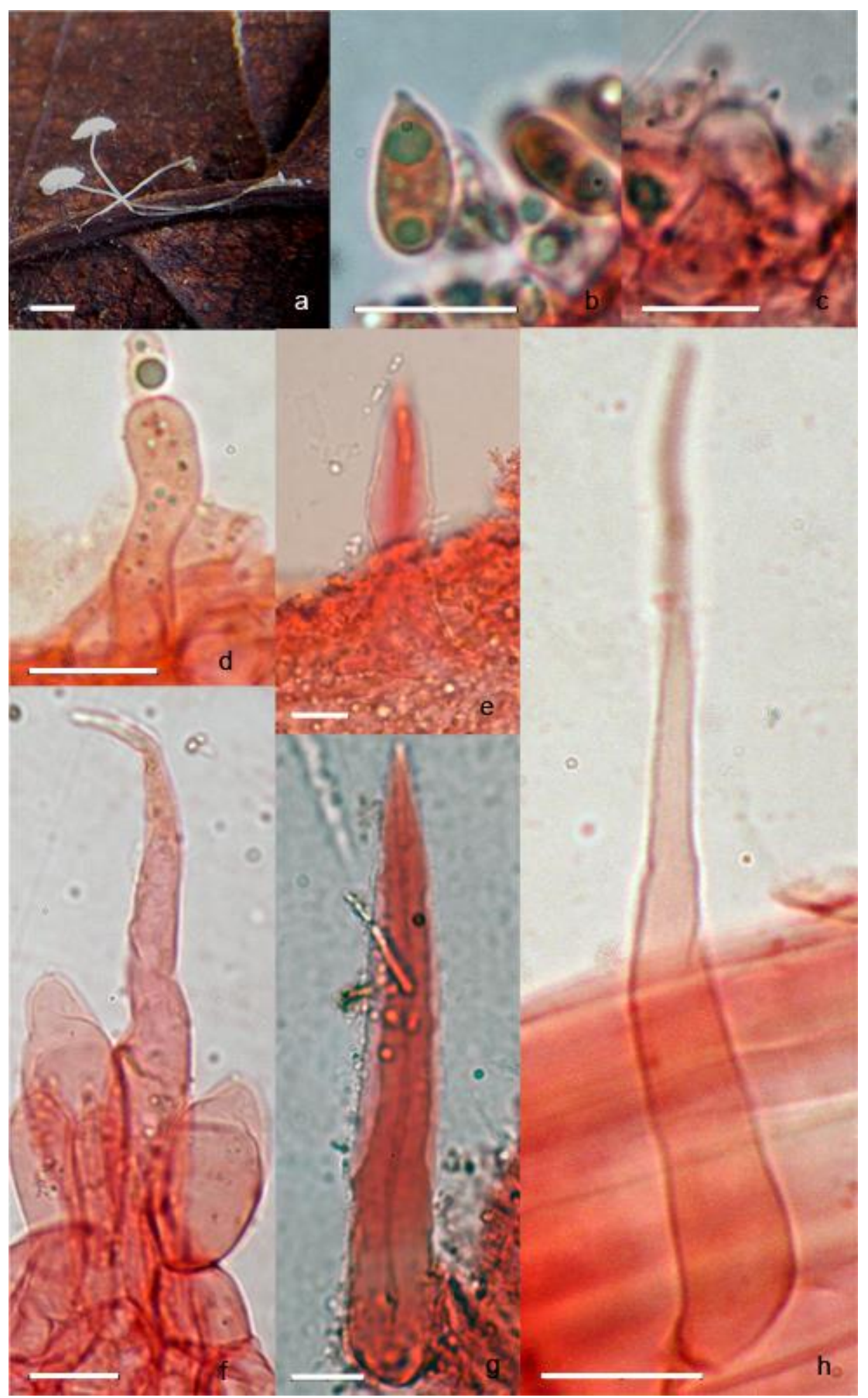

Fig. 3 a-h - Mycena pelava. a, Basidiomata. b, Basidiospore. c, Basidium. d, Cheilocystidium. e, g Pileosetae. f, Pileus marginal cells. h, Caulocystidium. - Bars $=5 \mathrm{~mm}$ for basidioma and $10 \mu \mathrm{m}$ for micro-structures.

up to 9 reaching the stipe, free, pure white, less than $0.5 \mathrm{~mm}$ wide, subdistant, with lamellulae of one length; edge very finely torn under a lens, concolorous with the sides. Stipe $4.5-5 \times 0.1-0.25$ $\mathrm{mm}$, central, terete, tapering towards the apex, hollow; surface translucent, white, with very fine, white pubescence, more so towards base, almost glabrous at the apex; base swollen, with fine, radiating basal mycelium. Context narrow, concolorous with the pileus surface. Odour and taste not recorded.

Basidiospores 7.5-8.5 (9) $\times 3-4.5(8.175 \pm 0.437 \times 3.85 \pm 0.366) \mu \mathrm{m}, \mathrm{Q}=1.78-2.67, \mathrm{Qm}=$ 2.14, oblong-ellipsoid to subcylindrical, thin-walled, hyaline, smooth, weakly amyloid. Basidia 10$15 \times 8-10 \mu \mathrm{m}$, subglobose to ovoid, hyaline, bearing 4 sterigmata up to $2.5 \mu \mathrm{m}$ long. Lamella-edge 
heterogeneous. Cheilocystidia scattered, 15.5-22 × 4.5-6 $\mu \mathrm{m}$, subcylindrical to flexuous, thinwalled, smooth. Pleurocystidia none. Lamellar trama subregular; hyphae 4-17 $\mu \mathrm{m}$, thin-walled, hyaline, faintly vinoid in Melzer's reagent. Subhymenium pseudoparenchymatous. Pileus trama subregular; hyphae 6-29 $\mu \mathrm{m}$ wide, thin-walled, hyaline, faintly vinoid in Melzer's reagent. Pileipellis a cutis of repent hyphae with terminal or intercalary pileosetae; hyphae $2-11 \mu \mathrm{m}$ wide, slightly gelatinized in $\mathrm{KOH}$, thin-walled, hyaline, with short, conic or cylindrical excrescences $(0.5-4 \times 0.5-1 \mu \mathrm{m})$. Pileosetae 45-215 × 6-22 $\mu \mathrm{m}$, aculeate, thick-walled $(1.5-6 \mu \mathrm{m})$, with a swollen, often geniculate base, slightly gelatinized in $\mathrm{KOH}$. Pileus marginal cells $27-91 \times 6-13.5$ $\mu \mathrm{m}$, clavate or fusiform, thin-walled, hyaline, with sparse, short, cylindrical excrescences $(0.5-4 \times$ $0.5-1 \mu \mathrm{m})$ mostly at the middle part or close to the apex. Stipitipellis a cutis with intercalary or terminal caulocystidia; hyphae 1.5-3 $\mu \mathrm{m}$ wide, thin-walled, hyaline, smooth. Caulocystidia 55-125.5 × 15-33 $\mu \mathrm{m}$, with a fusoid base and a long, aculeate apical part, thin-walled, hyaline. Stipe trama vinoid in Melzer's reagent. Clamp connections seen on all hyphae.

On decaying leaves, solitary or scattered, September.

Distribution - known only from the type locality.

Material examined - India, Kerala State, Thiruvananthapuram District, Palode, TBGRI Campus: 8 September 2009, D. M. Aravindakshan, K(M) 178342 (K-holotype).

Notes - This new species seems to be somewhat close to $M$. longiseta, a species restricted to Southeast Asia, as both species have aculeate pileosetae, convex, grey pileus and fusoid terminal cells of the pileus margin with spinulose lower portion and smooth acicular apices (see Desjardin \& Horak 2002). But the latter species has slightly larger basidiomata and non-gelatinized, longer (up to $1000 \mu \mathrm{m})$ pileosetae and lacks cheilocystidia. The present species seems to be close to $M$. brevisetosa as well in having somewhat similar sized basidiomata and basidiospores and fertile lamella-edge with smooth cheilocystidia. But that species differs in having corticolous basidiomata, a gelatinous layer in the pileal context, collariate lamellae, somewhat smaller (up to $130 \mu \mathrm{m}$ ) pileosetae, and a thick gelatinous layer on the pileus. Mycena saloma differs in having pileal marginal cells densely covered with verrucae, pileosetae without a swollen base, and caulocystidia with an obtuse apex and in lacking cheilocystidia.

Because the pileipellis is essentially a cutis made of repent, densely spinulose hyphae in all the three species described here, all of them can be placed in stirps Longiseta A.H. Sm. of sect. Longisetae. Apart from these three new species, two more species that belong to sect. Longisetae have already been described from Kerala State and all these species so far known from the region can be differentiated with the following key:

1. Basidiomata with brownish pileosetae; pileipellis a pulverulent epithelium composed of acanthocysts; cheilocystidia ventricose-fusoid with a spinulose lower middle region

M. lomavritha

1. Basidiomata with hyaline pileosetae; pileipellis a cutis composed of spinulose hyphae;

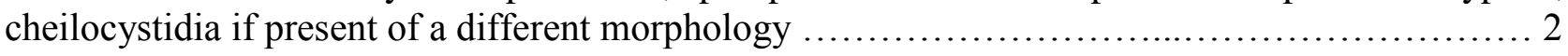

2. Caulocystidia thick-walled; lamellae pseudocollariate ......................... lomamaya

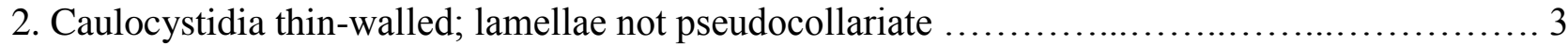

3. Cheilocystidia present, fusoid; pileosetae with excrescences at the base; basidiospores

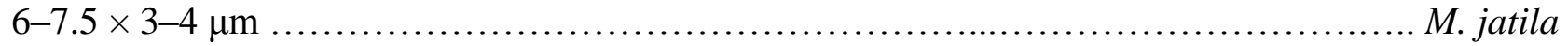

3. Cheilocystidia absent or if present not fusoid; pileosetae without excrescences at the base; basidiospores larger ...................................................................... 4

4. Cheilocystidia absent; pileal marginal cells densely covered with verrucae; pileosetae without a swollen base; caulocystidia with an obtuse apex; basidiospores 7.5-9.5 × 3.75-5.5 $\mu \mathrm{m}$ M. saloma

4. Cheilocystidia present; pileal marginal cells sparsely covered with verrucae; pileosetae with a swollen base; caulocystidia with an aculeate apex; basidiospores 7.5-8.5 $\times 3-4.5 \mu \mathrm{m}$ M. pelava 


\section{Acknowledgements}

One of us (DMA) acknowledges financial assistance from the University of Calicut in the form of a PhD fellowship.

\section{References}

Aravindakshan DM, Manimohan P. 2011- Mycena sect. Longisetae: a new species, a new name, and an addition. Mycotaxon 117, 239-246.

Aravindakshan DM, Manimohan P 2013 - A new section and two new species of Mycena. Mycosphere 4(5), 930-935.

Boonpratuang T. 2009 - Mycena variicystis, a new spinose species from Phru Toh Daeng Peat Swamp. Mycotaxon 109, 185-188.

Desjardin DE, Horak E. 2002 - Agaricales of Indonesia. 4. Mycena sect. Longisetae with comments on allied species. Sydowia 54, 142-156.

Desjardin DE, Boonpratuang T, Hywel-Jones N. 2002 - An accounting of the worldwide members of Mycena sect. Longisetae. Fungal Diversity 11, 69-85.

Kornerup A, Wanscher JH. 1978 - Methuen Handbook of Colour. 3rd Ed. Eyre Methuen, London.

Maas Geesteranus RA. 1983 - Conspectus of the Mycenas of the Northern Hemisphere-1. Sections Sacchariferae, Basipedes, Bulbosae, Clavulares, Exiguae, and Longisetae. Proceedings of the Koninklijke Nederlandse Akademie van Wetenschappen. Series C. Biological and medical sciences. 86, 401-421.

Maas Geesteranus RA, de Meijer AAR. 1998 - Further mycenas from the State of Paraná, Brazil. Persoonia 17, 29-46. 